\title{
Pharmacokinetics, Efficacy and Safety of a Plasma- Derived VWF/FVIII Concentrate (Formulation V) in Pediatric Patients with von Willebrand Disease (SWIFTLY-VWD Study)
}

This article was published in the following Dove Press journal:

Journal of Blood Medicine

\author{
Guenter Auerswald' \\ Claudia Djambas Khayat ${ }^{2}$ \\ Oleksandra Stasyshyn (iD) ${ }^{3}$ \\ Genadi losava $^{4}$ \\ Irina Romashevskaya ${ }^{5}$ \\ Marta Julia López ${ }^{6}$ \\ Wilfried Seifert ${ }^{7}$ \\ Tobias Rogosch (D) $^{7}$ \\ 'Hess Kinderklinik, Klinikum Bremen- \\ Mitte, Bremen, Germany; ${ }^{2}$ Hotel Dieu de \\ France Hospital, St Joseph University, \\ Beirut, Lebanon; ${ }^{3}$ Institute of Blood \\ Pathology and Transfusion Medicine, \\ Academy of Medical Sciences of Ukraine, \\ Lviv, Ukraine; ${ }^{4}$ Institute for Hematology \\ and Transfusiology, Tbilisi, Georgia; \\ ${ }^{5}$ Republican Research Centre for \\ Radiation Medicine and Human Ecology, \\ Gomel, Belarus; ${ }^{6} \mathrm{Hematology,} \mathrm{Hospital}$ \\ Roosevelt, Guatemala, Guatemala; \\ ${ }^{7}$ Clinical Development, CSL Behring, \\ Marburg, Germany
}

Correspondence: Guenter Auerswald Hess Kinderklinik, Klinikum BremenMitte, Bremen, Germany

Tel +49 I76-101 I 3362

Email guenterauerswald@aol.com
Purpose: Formulation V (VONCENTO ${ }^{\circledR}$ ) is a plasma-derived high-concentration/lowvolume, high-purity von Willebrand factor (VWF)/factor VIII (FVIII) concentrate, originally indicated for von Willebrand disease (VWD) in adults and adolescents. This multicenter, open-label study (SWIFTLY-VWD) evaluated the pharmacokinetics (PK), as well as hemostatic efficacy and safety, of Formulation $\mathrm{V}$ in pediatric patients $(<12$ years) with severe VWD requiring treatment or prophylaxis of bleedings.

Methods: PK investigations were performed following one dose of Formulation V at Day 1 and 180. Nonsurgical bleeds were analyzed, while hemostatic efficacy was graded as excellent/good/moderate/none. Safety assessments included adverse events, and presence of VWF and/or FVIII inhibitors.

Results: Formulation V was administered as on-demand $(\mathrm{N}=13)$ or prophylaxis therapy $(\mathrm{N}=4)$ for 12 months ( $<6$ years, $\mathrm{N}=9 ; 6$ to $<12$ years, $\mathrm{N}=8$ ). PK parameters for VWF markers were generally comparable to adults but showed lower VWF:ristocetin cofactor (RCo) exposure. Incidence of major bleeds was lower for prophylaxis $(3.3 \%)$ than on-demand therapy (27.1\%); joint bleeds were also lower (3.3\% vs $11.5 \%$, respectively). Investigatorreported excellent/good hemostatic efficacy against nonsurgical bleeds was $100 \%$. No clinically relevant differences in PK, hemostatic efficacy, or safety were observed between agegroups ( $<6$ years and 6 to $<12$ years). Formulation $\mathrm{V}$ was well tolerated. Adverse events were mild-moderate and consistent with the adult safety profile. No cases of anaphylactic reactions or angioedema, development of FVIII/VWF inhibitors, thromboembolic events, or viral infections were reported.

Conclusion: This study provides evidence for use of Formulation $\mathrm{V}$ to treat and prevent bleeding in pediatric patients with severe VWD, and led to the European approval of Formulation $\mathrm{V}$ in children.

Keywords: von Willebrand disease, clinical trial, pediatrics, prophylaxis, von Willebrand factor-factor VIII concentrate

\section{Introduction}

Von Willebrand disease (VWD) is an autosomal dominant or recessive inherited bleeding disorder resulting from an abnormality, either quantitative or qualitative, in the von Willebrand factor (VWF). ${ }^{1-4}$ The disease results from defects in the gene encoding VWF located at chromosome 12p13.2, and its severity is related to the type of gene defect. VWD is usually classified into three types (types 1-3) on the 
basis of clinical and laboratory phenotypes. Type 1, accounting for the large majority of cases (70-80\%), involves partial quantitative defects, while type 3 is characterized by a virtual absence of VWF in plasma. ${ }^{5,6}$ Type 2 variants consist of qualitative defects and can be subclassified into $2 \mathrm{~A}$ (lack of high- and intermediate-molecularweight multimers/decreased platelet function), 2B (lack of high-molecular-weight multimers/increased platelet function), 2M (normal multimer distribution/decreased platelet function), and $2 \mathrm{~N}$ (normal multimer distribution/decreased affinity for coagulation factor VIII [FVIII]). ${ }^{7}$

Clinically, the leading symptom in VWD is bleeding, mainly of mucosal origin (eg, epistaxis, gingival, or gastrointestinal bleeding, and heavy menstrual bleeding). The severe forms of VWD are characterized by greatly reduced levels of VWF activity (ie, VWF:ristocetin cofactor [VWF:RCo] of $<20$ international units [IU]/dL) and decreased FVIII:coagulant activity (FVIII:C) (ie, <20 IU/dL).

The aim of VWD therapy is to treat and prevent bleeding episodes due to abnormal platelet adhesion and blood coagulation, resulting from low or abnormal VWF and/or FVIII levels. ${ }^{7,8}$ Desmopressin acetate (1-deamino8-D-arginine vasopressin [DDAVP]) is the most widely used treatment for VWD, which induces the release of VWF from its endogenous storage location into the circulation. ${ }^{4}$ It is the preferred treatment for most patients with type $1 \mathrm{VWD}$, of whom $80 \%$ respond to DDAVP. ${ }^{8}$ In patients with types $1,2 \mathrm{~A}, 2 \mathrm{M}$, and $2 \mathrm{~N}$ VWD the response to DDAVP is variable, so it is suggested that a trial with DDAVP be conducted for each patient to determine efficacy. ${ }^{9}$ For patients who do not respond, all type 3 VWD patients and those in whom DDAVP is not sufficiently effective or contraindicated, VWF/FVIII concentrates derived from human plasma are recommended for both prophylaxis and treatment of bleeding. ${ }^{6,9}$

VONCENTO $^{\circledR}$; CSL Behring GmbH, Marburg, Germany (referred to here as Formulation V) is a plasmaderived, high-concentration, low-volume, high-purity VWF/FVIII concentrate, which contains a high level of high-molecular-weight VWF multimers and a VWF:RCo/ FVIII:C ratio of $\sim 2.4: 1$. Outside the EU, it is licensed and marketed by CSL Behring under the names BIOSTATE ${ }^{\circledR}$, ALEVIATE $^{\circledR}$, HUMAN COAGULATION FACTOR VIII $^{\circledR}$ or TBSF FACTOR ${ }^{\circledR} \cdot{ }^{10}$

Compared with Haemate $\mathrm{P}$ (referred to here as Formulation HP), a VWF/FVIII concentrate also manufactured by CSL Behring that has been available since 1981,
Formulation V has a similar VWF:RCo to FVIII:C ratio ${ }^{11}$ and multimeric profile. ${ }^{12}$ However, Formulation $\mathrm{V}$ has a higher concentration and lower volume, ${ }^{11}$ and different purification steps during manufacture (ie, heparin/glycine precipitation and gel chromatography compared with multiple precipitation for Formulation HP). ${ }^{4}$ The manufacture of Formulation $\mathrm{V}$ includes two dedicated viral inactivation steps, namely a solvent detergent treatment at an intermediate stage of the process and dry heat treatment of the freeze-dried product in the final container.

Formulation $\mathrm{V}$ has been evaluated in the Studies with von Willebrand factor/Factor VIII (SWIFT) program for the treatment of patients with hemophilia A or VWD across all ages, in accordance with European clinical and pediatric guidelines. SWIFT is a comprehensive clinical development plan across many European countries. To date, two studies in adults and adolescent patients ( $\geq 12$ years of age) have been published, one each in hemophilia A (SWIFTHA) and VWD (SWIFT-VWD). ${ }^{11,13}$ In the VWD study $(\mathrm{N}=21)$, excellent/good hemostatic efficacy was achieved in $98.3 \%$ of nonsurgical bleeding (NSB) events. ${ }^{11}$ Following the switch to prophylactic treatment in eight patients, the total number of NSB events was markedly reduced, from 304 to 10 (hemostatic efficacy was judged to be "excellent" for all 10). In terms of safety, no FVIII or VWF inhibitory antibodies were detected, no transmission of infectious agents occurred, and no thromboembolic events or treatment-related serious adverse events (SAEs) were reported.

The current study is the next in the SWIFT program, which extends the investigation to pediatric patients. Its aim was to evaluate the pharmacokinetics (PK) of Formulation $\mathrm{V}$ in children $<12$ years of age with severe VWD who required treatment or prophylaxis of bleedings. Hemostatic efficacy and safety were also investigated.

\section{Materials and Methods Study Design}

This Phase III, multicenter, open-label trial (NCT01213446; BIOSTATE $^{\circledR}$ tradename) was conducted in eight centers in Georgia, Germany, Guatemala, Lebanon (one center each), and Belarus and Ukraine (two centers each) between July 2010 and August 2013. Study participants included previously treated children $<12$ years old with severe type $1,2 \mathrm{~A}$, or 3 VWD (VWF:RCo $<20 \%$ at screening or a documented history of VWF:RCo $<10 \%$ ), for whom DDAVP treatment 
Table I Guidelines for Dosage During the Efficacy Component of the Study Were Adapted According to the Core SmPC for Hemophilia A and VWD [CPMP/BPWG/1619/1999;CPMP/BPWG/278/02], as Well as the Data Collected from Previous Efficacy Clinical Studies in WWD $28-30$

\begin{tabular}{|c|c|c|c|}
\hline Indication & $\begin{array}{l}\text { Dose (IU/kg bw) } \\
\text { VWF:RCo }\end{array}$ & Dose Frequency & Target FVIII : C/VWF :RCo (\%) \\
\hline \multirow[t]{2}{*}{ NSB events } & $25-50$ & Initial & VWF : RCo peak level >50\%, FVIII : C >30\% \\
\hline & 25 & Subsequent (every I2-24 h) & $\begin{array}{l}\text { VWF : RCo/FVIII : } C \text { trough levels of }>30 \% \text { until bleeding } \\
\text { stops (usually } 2-4 \text { days) }\end{array}$ \\
\hline Prophylaxis & $25-40$ & I-3 times weekly & Trough $>1 \%$ \\
\hline $\begin{array}{l}\text { Prophylaxis for } \\
\text { menorrhagia }\end{array}$ & $25-50$ & $\begin{array}{l}\text { On day I, days I and } 2 \text {, or days I, } 2 \\
\text { and } 3 \text { per cycle }\end{array}$ & VWF : RCo/FVIII : C peak levels $>30 \%$ \\
\hline Minor surgery & 60 & Daily & $\begin{array}{l}\text { VWF : RCo/FVIII : } C \text { trough levels of }>30 \% \text { until healing is } \\
\text { complete (usually } 2-4 \text { days) }\end{array}$ \\
\hline \multirow[t]{2}{*}{ Major surgery } & $60-80$ & Initial & VWF : RCo peak level $>100 \%$, FVIII : C >60\% \\
\hline & $30-60$ & Subsequent (every 12-24 h) & $\begin{array}{l}\text { VWF : RCo/FVIII : } C \text { trough levels of }>50 \% \text { until healing is } \\
\text { complete (usually } 5-10 \text { days) }\end{array}$ \\
\hline
\end{tabular}

Notes: Reproduced with permission from Lissitchkov TJ, Buevich E, Kuliczkowski K, Stasyshyn O, Cerqueira MH, Klukowska A, Joch C, Seifert W, Pharmacokinetics, efficacy, and safety of a plasma-derived VWF/FVIII concentrate (Formulation V) for on-demand and prophylactic treatment in patients with von Willebrand disease (SWIFTVWD study), Blood Coagul Fibrinolysis, 28, 2, 152-62,"' https://journals.Iww.com/bloodcoagulation/pages/default.aspx

Abbreviations: bw, body weight; FVIII:C, factor VIII:coagulant activity; IU, international unit; NSB, nonsurgical bleeding; SmPC, summary of product characteristics; VWD, von Willebrand disease; VWF:RCo, von Willebrand factor:ristocetin cofactor.

had been ineffective (criteria not specified in the protocol), contraindicated, or not available.

Participants required on-demand treatment of NSB events or were undergoing a surgical procedure, or were treated on a predefined prophylactic regimen. Patients with a known history or with suspected VWF or FVIII inhibitors were excluded. Ethics approval, written informed consent of the legal guardian, individual informed consent, and approval by the relevant national authorities were obtained prior to enrollment (Ethics Committee of the State Institution "Republican Scientific and Practical Center for Radiation Medicine and Human Ecology", Belarus; Joint Stock Company, The Institute of Hematology and Transfusiology, Georgia; Ethics Committee of the State of Bremen, Klinikum BremenMitte, Germany; Hospital Roosevelt Independent Ethics Committee, Guatemala; University of Saint-Joseph Beirut Ethics Committee, Lebanon; Ministry of Health of Ukraine Central Ethics Commission, Ukraine). This study adhered to the relevant Committee for Medicinal Products for Human Use (CHMP) guidelines. ${ }^{14}$ Where applicable, methods were similar to those in other SWIFT trials. ${ }^{11,13}$

Formulation V, containing either 1200 or 2400 international units [IU] VWF:RCo and 500 or 1000 IU FVIII:C per vial, respectively, was used in this study. The general dosing recommendation was 25-50 IU VWF:RCo/kg body weight (bw) for the treatment/prevention of spontaneous or trauma-induced hemorrhages and 60-80 IU VWF:RCo/ $\mathrm{kg}$ bw for minor or major surgery (Table 1). Each patient's treatment regimen and individual dose was determined by the investigator, based on the reason for use. Prior FVIII/ VWF therapy and exposure to VWF-containing products were recorded during the 12 months before the study. DDVAP or a VWF concentrate was not permitted in the 5 days before the first dose of Formulation V. All medication was recorded from 30 days before screening and throughout the study.

\section{Pharmacokinetics}

PK investigation was performed at Day 1 for all patients and at Day 180 for patients with type 3 VWD, who are the most susceptible to developing neutralizing antibodies (inhibitors) to VWF.

Initial and repeat PK analysis was performed following a single intravenous bolus dose of $80 \mathrm{IU}$ VWF:RCo/ $\mathrm{kg}$ bw (administered at a maximum infusion speed of $6 \mathrm{~mL} / \mathrm{min}$ ). Blood samples were collected for PK evaluation immediately prior to dosing with Formulation $\mathrm{V}$, and at $0.5,4,8,24$, and 48 hours after the infusion. PK parameters were calculated by 
noncompartmental analysis based on baseline-corrected concentrations. The following PK assays were performed and have been described previously: ${ }^{11}$ for VWF:RCo, a platelet agglutination method in the presence of $\mathrm{RCo}$; for VWF: collagen binding (CB) and VWF:antigen (Ag), enzymelinked immunosorbent assays (ELISAs); and for FVIII:C, a chromogenic FVIII:C assay. Professor Ulrich Budde (Medilys Laborgesellschaft, Asklepios Institut, Hamburg, Germany) performed and validated all assays. The lower limit of quantitation was $0.1 \mathrm{IU} / \mathrm{mL}$ (VWF:RCo), $0.025 \mathrm{IU} /$ mL (VWF:Ag;VWF:CB) and 0.008 IU/mL (FVIII:C), respectively. VWF:RCo activity plasma levels were used as a surrogate parameter for inhibitor development to trigger further testing for VWD inhibitors.

\section{Efficacy Assessments}

The number and classification of bleeding events (major or minor) were recorded in the 12 months before the study for both on-demand and prophylactic treatment. Hemostatic efficacy analysis was performed throughout the 12-month study duration. The clinical efficacy parameters assessed in the study were Formulation $\mathrm{V}$ usage, hemostasis assessment (overall every 3 months by the investigator, and for each NSB and surgical event by the investigator and patient), blood product transfusion requirements, and surgeon's assessment of blood loss during a surgical procedure ("less", "equivalent", or "more" compared with the expected blood loss from a patient without a bleeding disorder undergoing the same procedure). Clinical assessments of hemostatic efficacy were based on a four-point efficacy grading scale: "excellent" if hemostasis was achieved/cessation of bleeding occurred; "good" if slight oozing or partial but adequate control of bleeding occurred and no additional product was required for unplanned treatment; "moderate" if moderate bleeding or moderate control of bleeding occurred and additional product was required for unplanned treatment; and "none" in cases of severe uncontrolled bleeding. ${ }^{11}$ During the study, the investigator assessed the severity of NSB events as major or minor. Major NSB events included any bleeding into a joint/muscle, or a mucosal bleeding of the gastrointestinal tract (but not oral or nasal bleeding). All other NSB events were rated as minor, unless otherwise specified by the investigator.

For investigation of prophylactic treatment, patients performed a daily assessment of each NSB event and an overall monthly assessment. Additionally, an overall hemostatic efficacy assessment was performed by the investigator, based on Formulation $\mathrm{V}$ usage in the previous 3 months, the number of NSB events that occurred during this time, and additional treatment needed.

\section{Safety}

The safety analysis included all patients who had received at least one dose of Formulation V. Safety assessments included SAEs, the presence of VWF and/or FVIII inhibitors, laboratory parameters (eg, biochemistry, hematology, and urinalysis), a physical examination, and assessment of vital signs.

PK parameters, VWD phenotype, presence of FVIII and VWF inhibitors, and seroconversion for virus markers (indicative of hepatitis A virus [HAV], hepatitis B virus [HBV], hepatitis $\mathrm{C}$ virus [HCV], and human immunodeficiency virus [HIV] infection) were assessed or conducted in a central laboratory (Professor Ulrich Budde; Medilys Laborgesellschaft, Asklepios Institut, Hamburg, Germany). The presence of FVIII inhibitors was analyzed by the Bethesda method (Nijmegen modification). The presence of VWF inhibitors was analyzed by a Bethesda method-based assay with VWF:Ag and VWF:CB. Patients who were HAVand $\mathrm{HBV}$-negative were vaccinated against these infections before the first dose of Formulation V. Virology reference samples were taken at Day 1 and the final visit, though they were analyzed only if considered necessary.

\section{Statistical Procedures}

Primary endpoints were: PK parameters for VWF and FVIII following the first dose of Formulation $\mathrm{V}$ for all patients and after 6 months in Type 3 VWD patients; subjective assessment of hemostatic efficacy following a NSB event, surgical procedure or during prophylaxis use; hemostasis during surgical procedure; blood loss; and transfusion needs. Secondary endpoints were related to adverse events (AEs), and VWF and FVIII inhibitor development. Descriptive statistics were used to summarize continuous variables (mean and standard deviation, and/or median and range). Categorical variables are presented as numbers and percentages in frequency tables. Summaries are based on observed data (missing data were not replaced). Formal statistical tests were not performed.

\section{Results}

\section{Patients}

A total of 17 pediatric patients (7 type 2A; 10 type 3 ) were enrolled; four participated in the prophylaxis arm, and 13 


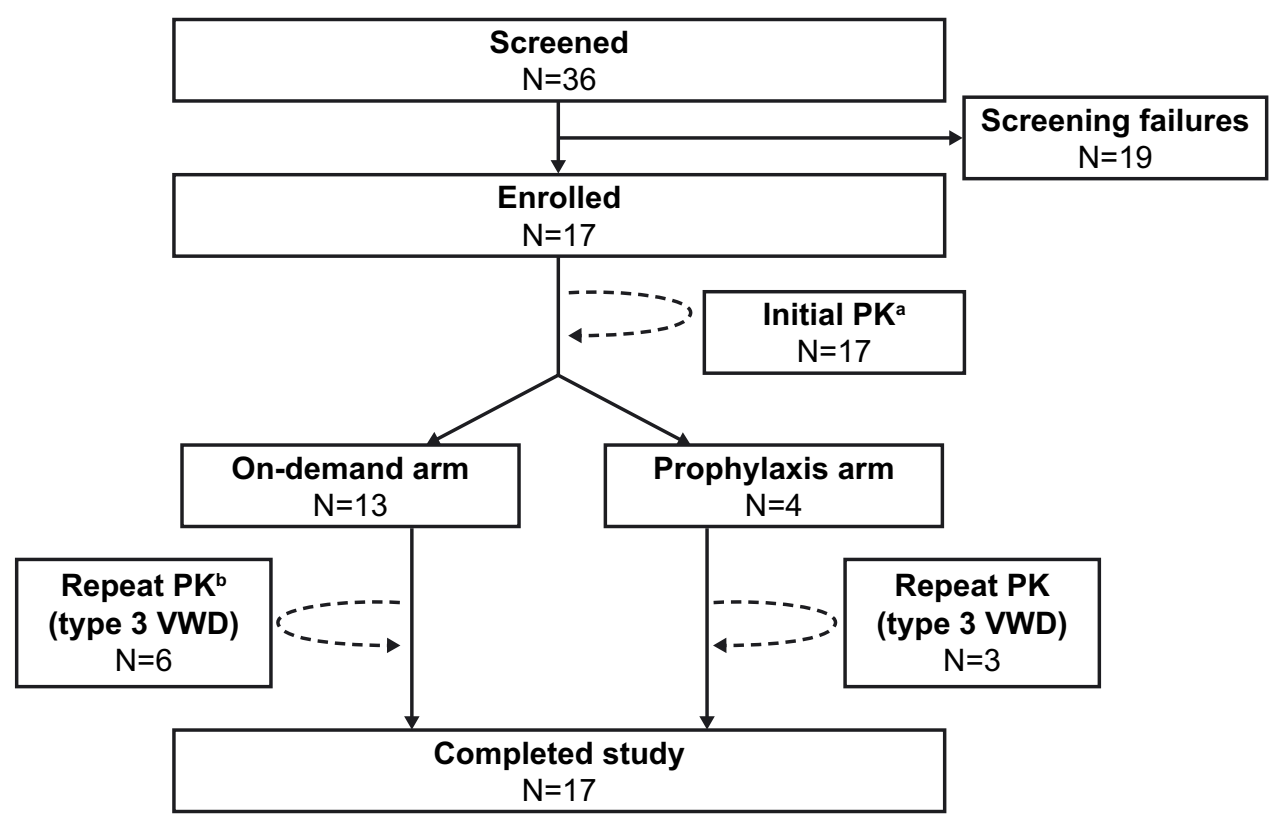

Figure I Patient disposition.

Notes: ${ }^{a}$ I 4 subjects eligible for analysis. ${ }^{b} 5$ subjects eligible for analysis.

Abbreviations: PK, pharmacokinetics; VWD, von Willebrand disease.

in the on-demand arm. Although patients with type 1 VWD were allowed in the study, none were actually enrolled. The patient disposition is shown in Figure 1.

Baseline patient characteristics are listed in Table 2. All patients completed the study. The duration of study participation for these patients ranged from 12 to 13 months. In the year preceding study entry, all patients in the on-demand arm and one patient in the prophylaxis arm had been treated on-demand, while the other three subjects in the prophylaxis arm had received prophylactic regimens.

\section{Pharmacokinetics}

PK parameters were assessed in all eligible patients $(n=14)$ after an initial infusion of Formulation V ( 3 of the 17 enrolled patients were excluded due to major PK deviations [dose calculation errors]) (Table 3). Nine of the patients in the initial PK analysis group underwent repeat PK evaluation; eight were eligible for PK analysis. Three patients in the initial PK group and one patient in the repeat PK group were excluded from analysis due to major protocol deviations (the dose calculation was erroneously based on FVIII:C instead of VWF:RCo, which led to administration of doses higher than the prescribed study dose).

Following Formulation V infusion, plasma concentrations of all VWF markers increased immediately, reached maximum plasma concentration $\left(\mathrm{C}_{\max }\right)$ at 30 min post-dose, and then declined over time with median terminal half-life $\left(t_{1 / 2}\right)$ of
10.0-13.5 $\mathrm{h}$ and median residence time (MRT) of 11.7-16.7 h. Concentration-time curves showed a similar profile of concentrations over time for all patients, and mean adjusted plasma concentrations for VWF:RCo were lower than for VWF:Ag and VWF:CB (Figure 2). VWF:CB/VWF:Ag, VWF:RCo/VWF:Ag and FVIII:C/VWF:Ag ratios are shown in Supplemental Figure 1. Based on baseline-adjusted data, median incremental recovery (IR) for VWF markers was $0.012-0.016(\mathrm{IU} / \mathrm{mL}) /(\mathrm{IU} / \mathrm{kg})$, median total clearance (CL) was 4.9-7.3 mL/h/kg, and median volume of distribution at steady state $\left(\mathrm{V}_{\mathrm{SS}}\right)$ was $68-112 \mathrm{~mL} / \mathrm{kg}$ (Table 3$)$.

Median $\mathrm{C}_{\max }$ of FVIII was $0.74 \mathrm{IU} / \mathrm{mL}$ (range: $0.33-1.67$ $\mathrm{IU} / \mathrm{mL}$ ) and FVIII levels were within the normal range. Compared with VWF PK, FVIII:C had a higher IR (0.018$0.020[\mathrm{IU} / \mathrm{mL}] /[\mathrm{IU} / \mathrm{kg}])$ and longer $\mathrm{t}_{1 / 2}(11.4-19.5 \mathrm{~h})$ (Table 3 ). It should be noted that VWD patients synthesize FVIII normally and their FVIII:C replacement kinetics reflect cumulative exogenous (infused) as well as endogenous (synthesized) FVIII, leading to longer $\mathrm{t}_{1 / 2}$ compared with untreated subjects. Both exogenous and endogenous FVIII bind to exogenous VWF.

Following repeated Formulation V administration for PK at Day 180, mean plasma concentrations for all analytes were comparable with those from the initial treatment (Figure 2). VWF:CB/VWF:Ag, VWF:RCo/VWF:Ag and FVIII:C/ VWF:Ag ratios are shown in Supplemental Figure 1. In general, IR, $\mathrm{C}_{\max }$, area under the curve (AUC), $\mathrm{t}_{1 / 2}$, and 
Table 2 Patients' Characteristics at Baseline

\begin{tabular}{|c|c|c|c|}
\hline Variable & $\begin{array}{l}\text { Prophylaxis } \\
\mathrm{N}=4\end{array}$ & $\begin{array}{l}\text { On-Demand } \\
N=13\end{array}$ & Overall $\mathrm{N}=17$ \\
\hline \multicolumn{4}{|l|}{$\mathrm{Age}^{\mathrm{a}}$} \\
\hline$<6$ years, $\mathrm{n}(\%)$ & $3(75.0)$ & $6(46.2)$ & $9(52.9)$ \\
\hline 6 to $<12$ years, $\mathrm{n}(\%)$ & I (25.0) & $7(53.8)$ & $8(47.1)$ \\
\hline Mean (SD), years & $5.0(2.5)$ & $5.3(3.7)$ & $5.2(3.4)$ \\
\hline Median (range), years & $5.0(2-8)$ & $6.0(0-11)$ & $5.0(0-11)$ \\
\hline \multicolumn{4}{|l|}{ Sex, n (\%) } \\
\hline Male & $4(100.0)$ & $3(23.1)$ & $7(41.2)$ \\
\hline Female & 0 & $10(76.9)$ & $10(58.8)$ \\
\hline \multicolumn{4}{|l|}{ Ethnic Origin, n (\%) } \\
\hline Caucasian & $4(100.0)$ & II (84.6) & $15(88.2)$ \\
\hline Black & 0 & I (7.7) & I (5.9) \\
\hline Hispanic & 0 & I (7.7) & I (5.9) \\
\hline \multicolumn{4}{|l|}{ Weight, $\mathrm{kg}^{\mathrm{a}}$} \\
\hline Mean (SD) & $20.4(8.2)$ & $22.3(14.1)$ & $21.9(12.7)$ \\
\hline Median (range) & $18.2(13.0-32.0)$ & $21.0(7.2-62.0)$ & $18.6(7.2-62.0)$ \\
\hline \multicolumn{4}{|l|}{ Height, $\mathrm{cm}^{\mathrm{a}}$} \\
\hline Mean (SD) & II $3.3(22.8)$ & II $0.4(24.8)$ & III.I (23.7) \\
\hline Median (range) & $109.5(90-144)$ & $108.0(67-152)$ & $108.0(67-152)$ \\
\hline \multicolumn{4}{|l|}{ VWD Type, n (\%) } \\
\hline Type I & 0 & 0 & 0 \\
\hline Type 2A & I (25.0) & $6(46.2)$ & $7(4 \mid .2)$ \\
\hline Type 3 & $3(75.0)$ & $7(53.8)$ & $10(58.8)$ \\
\hline \multicolumn{4}{|l|}{$\begin{array}{l}\text { VWD-Related Products } \\
\text { in the Previous } \\
12 \text { Months, } \mathrm{n}(\%)^{\mathrm{b}}\end{array}$} \\
\hline VWF/FVIII & $3(75.0)$ & $8(61.5)$ & II (64.7) \\
\hline FVIII & I (25.0) & $2(15.4)$ & $3(17.6)$ \\
\hline Anti-hemophilic factor & 0 & $3(23.1)$ & $3(17.6)$ \\
\hline
\end{tabular}

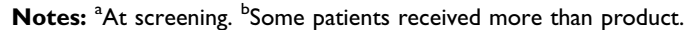

Abbreviations: FVIII, factor VIII; $N$, total number of patients; $n$, number of patients with characteristics; SD, standard deviation; VWD, von Willebrand disease; VWF, von Willebrand factor.

other PK parameters were comparable with those after the initial treatment, without signs of decreased AUC or accelerated elimination (Supplemental Table 1).

PK parameters for VWF:Ag and VWF:CB (eg, IR and $\left.t_{1 / 2}\right)$, were generally similar between age groups $(<6$ years and 6 to $<12$ years) in both the initial (Table 3 ) and repeat analyses. However, $t_{1 / 2}$ and mean residence time (MRT) were longer for younger children in the initial analyses, but shorter in the repeat analysis.

\section{Hemostatic Efficacy On-Demand Arm}

One patient in the on-demand arm did not experience any bleeding events during the study and was therefore excluded from all efficacy analyses. The 12 patients who

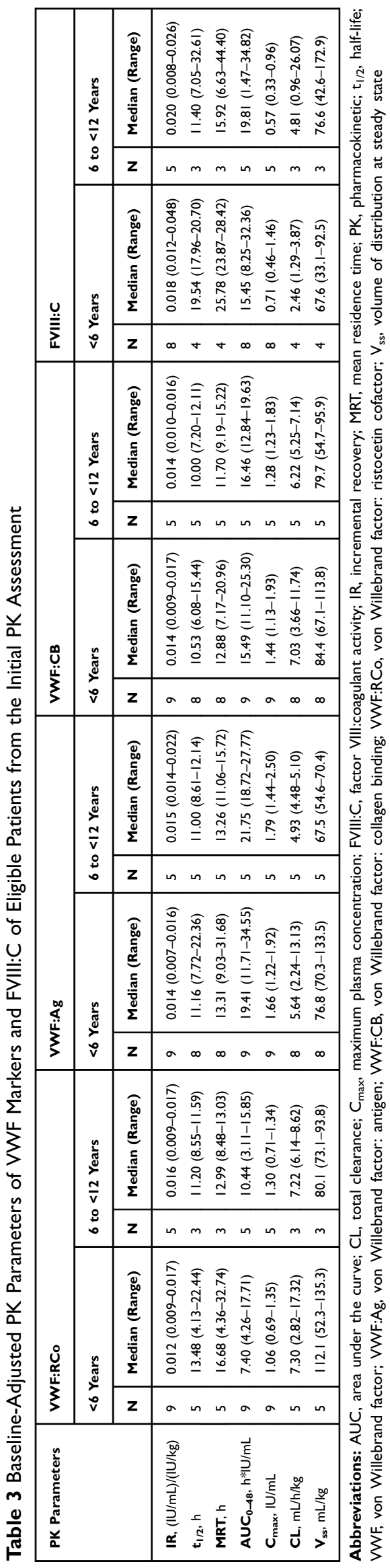



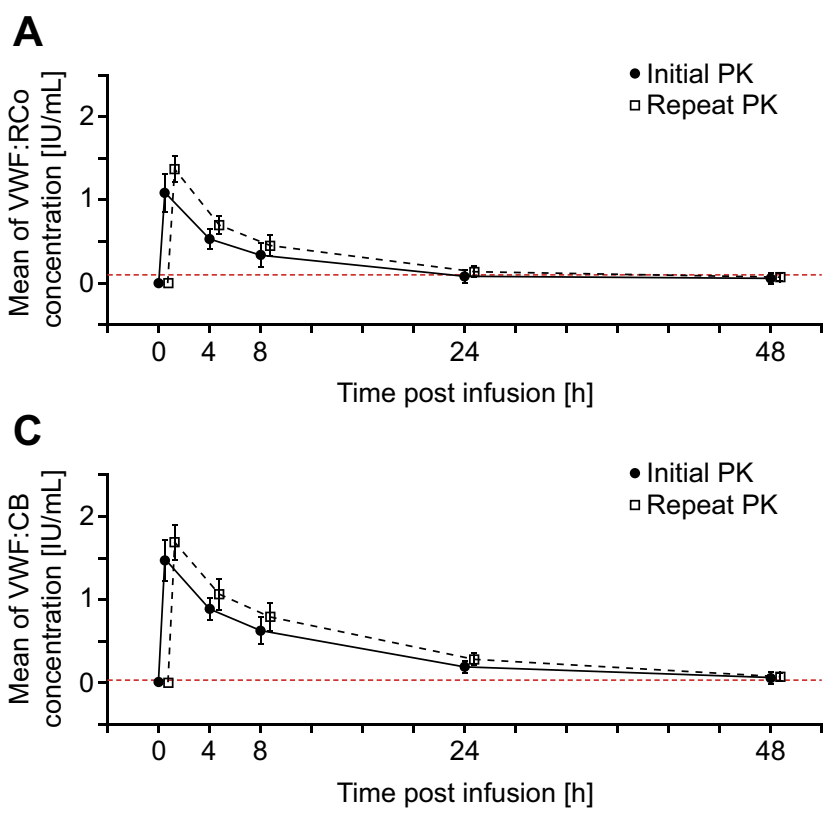

B

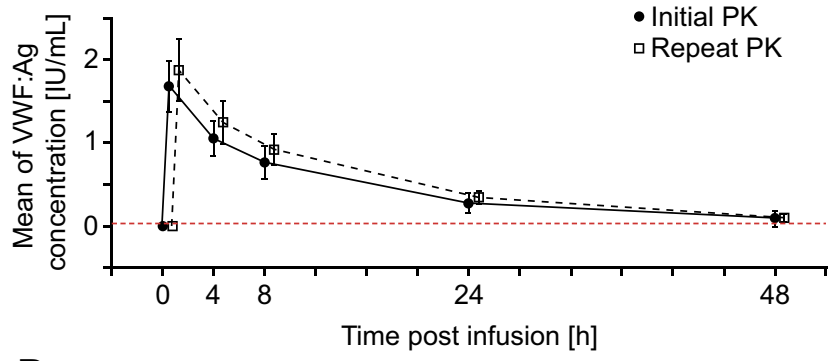

D

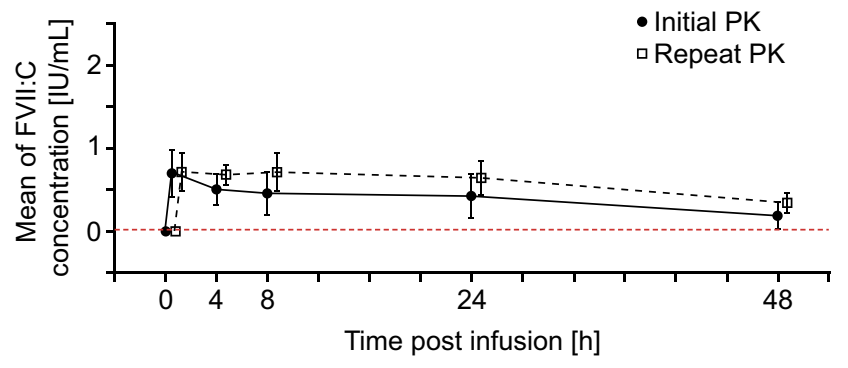

Figure 2 Mean (SD) concentration profiles (IU/mL) of baseline-adjusted VWF:RCo, VWF:Ag, VWF:CB, and FVIII:C in patients below the age of I2 (initial PK [N=I4], repeat PK [N=8]). Red dashed line: lower limit of quantitation (VWF:RCo: $0.1 \mathrm{IU} / \mathrm{mL} ; \mathrm{VWF}: \mathrm{Ag}, \mathrm{VWF}: \mathrm{CB}: 0.025 \mathrm{IU} / \mathrm{mL} ; \mathrm{FVIII:C:} 0.008 \mathrm{IU} / \mathrm{mL}$ ).

Abbreviations: FVIII:C, factor VIII:coagulant activity; PK, pharmacokinetics; SD, standard deviation; VWF:Ag, von Willebrand factor: antigen; VWF:CB, von Willebrand factor: collagen binding; VWF:RCo, von Willebrand factor: ristocetin cofactor.

were treated on-demand experienced a total of $96 \mathrm{NSB}$ events, resulting in a median of 5.5 NSB events per patient, with a range of 1-22.

A total of $80 \mathrm{NSB}$ events $(83.3 \%)$ required treatment with Formulation V, while 16 (16.7\%) did not. The majority of all bleeding events required only one infusion of Formulation V (57.3\%), while 25 NSB events (26.0\%) required a median of two infusions (range: 2-8). Patients had a median Formulation V dose of 43.6 IU VWF:RCo/ $\mathrm{kg}$ bw (range: 34.2-66.1 IU VWF:RCo/kg bw). Of the medicated NSB events, $51(63.8 \%)$ were treated at home and $29(36.3 \%)$ were treated in hospital.

Excellent or good hemostatic efficacy was reported by the investigator for all 80 medicated NSB events (Table 4 and Figure 3A). Additionally, hemostatic efficacy was assessed by patients/legal guardians as excellent for $60.0 \%$ of the 55 assessed bleeding days, good for $21.8 \%$, and moderate for $18.2 \%$. Hemostatic efficacy for the patients $<6$ years old (excellent $48.5 \%$; good 51.5\%) in the on-demand arm was similar to that seen in the patients 6 to $<12$ years old (excellent 42.6\%; good 57.4\%).

Total number of minor and major bleeding events, and locations of bleeds in the on-demand arm are shown in Table 4 (treated NSB events), and Figure $3 \mathrm{~B}$ and $\mathrm{C}$ (all NSB events). The majority of all NSB events were considered minor (72.9\%), and were mucosal $(82.3 \%)$. A total of 26 treated NSB events were considered major, including 13 mucosal and 11 joint events. The average annualized bleeding rate (ABR) was 5.5 minor bleeds per year and 2.0 major bleeds per year.

\section{Prophylaxis Arm}

The four patients in the prophylaxis arm reported a total of $91 \mathrm{NSB}$ events, resulting in a median of 23.5 NSB events per patient, with a range of 9-35. Patients experienced 9, 14, 33, and 35 NSB events, respectively.

A total of $73(80.2 \%)$ NSB events required treatment with Formulation V, while 18 (19.8\%) did not. The majority of all NSB events required only one infusion of Formulation $\mathrm{V}(78.0 \%)$, while two $(2.2 \%)$ required a median of 7 infusions (range: 3-11). Patients received a median number of 119 infusions (range: 45-161) at a median Formulation $\mathrm{V}$ dose of 61.6 IU VWF:RCo/kg bw (range: 46.0-66.1 IU VWF: $\mathrm{RCo} / \mathrm{kg} \mathrm{bw}$ ) for prophylaxis, and a median dose of $62.8 \mathrm{IU}$ VWF:RCo/kg bw (range: 45.5-74.6 IU VWF:RCo/kg bw) to treat NSB events.

Excellent or good hemostatic efficacy was reported by the investigator for all 73 medicated NSB events (Table 4 and Figure 3A). Moreover, for each 3-month interval, prophylactic hemostatic efficacy was assessed retrospectively by the investigator as either excellent or good. The patient/legal guardian 
Table 4 Investigator's Assessment of Hemostatic Efficacy per Treated NSB Events and Overall Prophylactic Efficacy

\begin{tabular}{|c|c|c|c|c|c|c|c|c|}
\hline \multirow[t]{4}{*}{ Bleeding Type } & \multicolumn{8}{|c|}{ Hemostatic Efficacy Assessment of Treated NSB Events } \\
\hline & \multicolumn{4}{|c|}{ On-Demand $(\mathrm{N}=12)$} & \multicolumn{4}{|c|}{ Prophylaxis $(\mathrm{N}=4)$} \\
\hline & \multicolumn{4}{|c|}{ Number (\%) of NSB Events } & \multicolumn{4}{|c|}{ Number (\%) of NSB Events } \\
\hline & $\mathbf{n}$ & Excellent & Good & Moderate & $\mathbf{n}$ & Excellent & Good & Moderate \\
\hline All bleedings & 80 & $36(45.0)$ & $44(55.0)$ & - & 73 & $59(80.8)$ & $14(19.2)$ & - \\
\hline Spontaneous & 62 & $26(41.9)$ & $36(58.1)$ & - & 59 & $49(83.1)$ & $10(16.9)$ & - \\
\hline Trauma & 18 & $10(55.6)$ & $8(44.4)$ & - & 14 & $10(71.4)$ & $4(28.6)$ & - \\
\hline Post-surgery & 0 & - & - & - & 0 & - & - & - \\
\hline Major & 26 & $13(50.0)$ & $13(50.0)$ & - & 3 & I (33.3) & $2(66.7)$ & - \\
\hline Minor & 54 & $23(42.6)$ & 31 (57.4) & - & 70 & $58(82.9)$ & $12(17.1)$ & - \\
\hline Joint & 11 & $2(18.2)$ & $9(81.8)$ & - & 3 & - & $3(100)$ & - \\
\hline Mucosal & 65 & $30(46.2)$ & $35(53.8)$ & - & 62 & $53(85.5)$ & $9(14.5)$ & - \\
\hline Muscle & 1 & I (100) & - & - & 0 & - & - & - \\
\hline Other & 3 & $3(100)$ & - & - & 8 & $6(75.0)$ & $2(25.0)$ & - \\
\hline \multirow[t]{3}{*}{ Visit } & \multicolumn{8}{|c|}{ Overall Hemostatic Efficacy Assessment of Prophylaxis } \\
\hline & & & & & \multicolumn{4}{|c|}{ Number (\%) of Patients } \\
\hline & - & - & - & - & $\mathbf{N}$ & Excellent & Good & Moderate \\
\hline Month 3 & - & - & - & - & 4 & $2(50.0)$ & $2(50.0)$ & - \\
\hline Month 6 & - & - & - & - & 4 & $4(100)$ & - & - \\
\hline Month 9 & - & - & - & - & 4 & $4(100)$ & - & - \\
\hline Month 12 & - & - & - & - & 4 & $3(75.0)$ & I (25.0) & - \\
\hline
\end{tabular}

Note: Bleedings with missing investigator's assessment for efficacy and those for which no treatment was needed are not included.

Abbreviations: $N$, total number of patients; n, number of treated bleeding events; NSB, non-surgical bleeding.

assessment of hemostatic efficacy for the 76 assessed bleeding days (excellent [78.9], good [21.1]) was generally in line with the investigator's assessment (excellent [80.8], good [19.2]). Hemostatic efficacy for the three patients $<6$ years old was excellent $(75.0 \%)$ or good $(25.0 \%)$ in the prophylaxis arm; corresponding values for the one patient $>6$ years old were $90.3 \%$ and $9.7 \%$, respectively.

The total number of minor and major bleeding events, and locations of bleeds in the prophylaxis arm are shown in Table 4, and Figure 3B and C. The majority of all NSB events were considered minor (96.7\%), and were mucosal (87.9\%). Three patients experienced one major joint NSB event each. The ABR was 22.0 minor bleeds per year and 0.7 major bleeds per year.

The mean (and median) number of NSB events in the 12 months before the study was 14 (range: 2-27), while during the study a mean of 22.8 (median: 23.5) NSB events were experienced. However, when the type (or category) of NSB events is examined, the rate of major NSB events was reduced from $9.3 \%$ before the study to $3.3 \%$. In addition, all major and minor NSB events were treated effectively (excellent $33.3 \%$ and $82.9 \%$, good $66.7 \%$ and $17.1 \%$, respectively) (Table 4). During the study, the incidence of major bleeds was lower in patients on prophylaxis $(3.3 \%)$ than on-demand therapy (27.1\%); joint bleeds were also lower (3.3\% vs $11.5 \%$, respectively).

\section{Surgical Events}

During the study, three patients in the on-demand arm underwent a total of eight minor surgeries: four tooth extractions and four surgeries denoted as tooth care. All surgical events were treated with one infusion of Formulation V prior to the surgery; one tooth extraction was additionally treated with one infusion during surgery and two infusions after surgery.

The investigator's assessment of hemostatic efficacy was assessed as excellent for seven surgical events (87.5\%) and as good for one event (12.5\%). Blood loss during surgery was assessed as comparable to the expected blood loss from a patient without a bleeding disorder undergoing the same procedure in all surgical events. No patient required blood product transfusions during the entire study. 
A

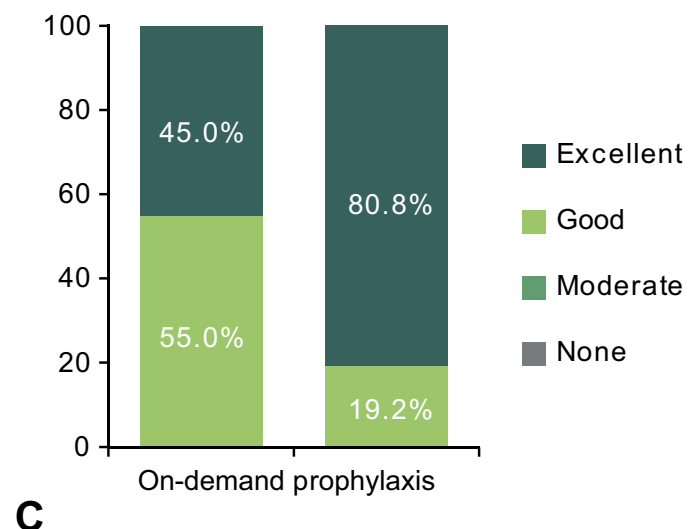

B

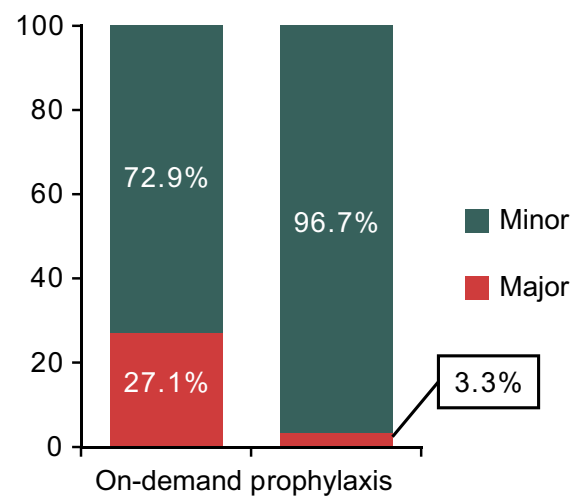

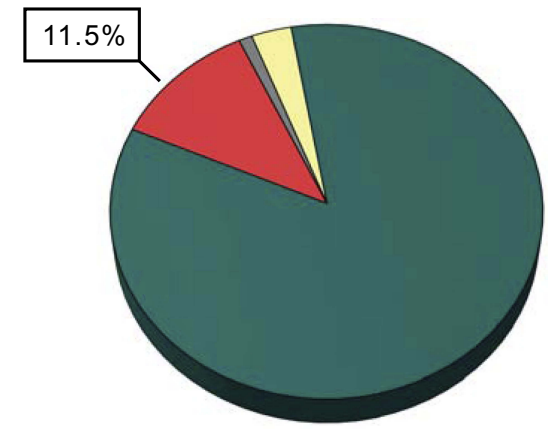

On-demand

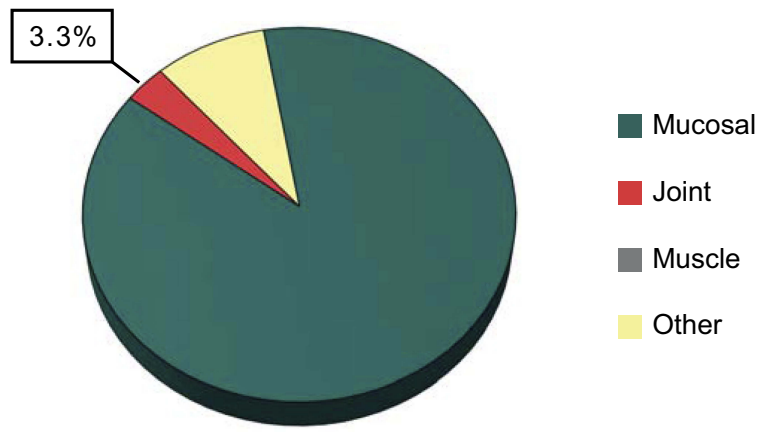

Prophylaxis

Figure 3 Investigator's efficacy assessment of treated bleeding events (A), minor and major bleeding events (including bleeding events without treatment) (B), and locations of bleeds (including bleeding events without treatment) $(\mathbf{C})$ in both treatment arms.

\section{Safety}

The median total Formulation $\mathrm{V}$ dose received by the four patients in the prophylaxis arm was $8062 \mathrm{IU}$ VWF:RCo/kg bw (range: 3244-13,642 IU VWF:RCo/kg bw) over a median of 129 exposure days (range: 55-197 days); 536 IU VWF:RCo/kg bw (range: 80-2080 IU VWF: $\mathrm{RCo} / \mathrm{kg}$ bw) was received over 8 exposure days (range: 1-36 days) by the 13 subjects in the on-demand arm.

Overall, Formulation V was well tolerated. During this study, twelve patients $(70.6 \%)$ reported a total of 77 treatment-emergent adverse events (TEAEs): three patients (75.0\%) in the prophylaxis arm had 40 TEAEs, and nine patients $(69.2 \%)$ in the on-demand arm had 37 TEAEs. TEAEs were generally mild to moderate in intensity. Three patients reported a total of four TEAEs of severe intensity that were considered to be not related to Formulation V (gastroenteritis, stomatitis, joint injury, and clavicle fracture). The most frequently reported TEAEs were pyrexia (6 patients [35.3]), rhinitis (4 patients [23.5]), and cough (4 patients [23.5]). No TEAEs leading to discontinuation of Formulation $\mathrm{V}$ and no serious TEAEs or TEAEs of special interest (anaphylactic reactions/ angioedema, development of VWF/FVIII inhibitors, virus transmissions, and thromboembolic events) occurred during this study. Two patients (one in each arm) had one TEAE each (infusion site erythema and pyrexia, respectively) that was considered at least possibly related to Formulation V. Both events were mild in intensity, nonserious, and had resolved on the same day the events started.

There were no clinically relevant differences in the TEAE reporting profile between patients aged $<6$ years and those aged 6 to $<12$ years. Reported TEAEs were characteristic for these age groups.

Two patients in the prophylaxis arm had low postbaseline hemoglobin values that were considered clinically significant by the investigator, but similar values were reported at baseline. One of these patients had an abnormally low mean corpuscular volume and abnormally high alanine aminotransferase and aspartate aminotransferase values, reflecting the patient's medical history of anemia, increased transaminases, and lymphadenopathy at study entry (not considered by the investigator to have affected the conduct 
of the study). None of these abnormal laboratory values were considered related to Formulation V. No other clinically relevant findings were seen in the analyses of physical examinations, vital signs, or body weight.

At all timepoints where VWF and FVIII inhibitors were assessed, all patients had a negative inhibitor titer.

\section{Discussion}

This study investigated the PK, as well as the efficacy and safety, of Formulation $\mathrm{V}$ in children up to 12 years of age with severe VWD. The data formed the basis of the submission to the European Medicines Agency in September 2014, who subsequently approved Formulation $\mathrm{V}$ for pediatric patients in June 2015, and it is now approved in all ages for the prophylaxis and treatment of hemorrhage or surgical bleeding in patients with VWD, where DDVAP alone is not effective or is contraindicated.

The PK results from this study were generally comparable with those in adult VWD patients. However, pediatric patients showed lower VWF:RCo exposure than adults, as indicated by a lower IR, shorter $t_{1 / 2}$ and faster CL. VWF:Ag, VWF:CB and FVIII:C PK showed similar trends. Based on these results (higher volumes of distribution and faster clearance as known for almost all factor concentrates used for replacement therapy), a prophylactic dose range of 40-80 IU VWF:RCo/kg bw 1 to 3 times a week should be considered in patients under 12 years of age. For long-term prophylaxis in adult and adolescent patients with VWD, a lower dose of 25-40 IU VWF: $\mathrm{RCo} / \mathrm{kg}$ bw has been shown to be sufficient at a frequency of 1 to 3 times per week. ${ }^{10}$ Formulation V exposure and disposition were generally comparable between $<6$-year-old and 6 to $<12$-year-old patients.

PK parameters for VWF markers from the repeat PK evaluation of this study were similar to those from initial PK analysis. The consistency of these data suggests that no inhibitors for VWF:RCo, VWF:Ag, VWF:CB, and FVIII:C developed after repeated treatment with Formulation $\mathrm{V}$ for 6 months.

A number of plasma-derived concentrates are used to treat VWD, with a recombinant VWF recently available (VONVENDI $^{\circledR}$; Shire, Inc., Cambridge, MA, USA). ${ }^{7,15-18}$ Use of $\operatorname{VONVENDI}^{\circledR}$, however, is currently restricted to adults. ${ }^{18}$ Though two Phase three open-label pediatric studies are underway (NCT02932618 and NCT03879135) no data are yet available. VWF/FVIII concentrates have similar hemostatic efficacy and PK, but Formulation HP is widely used in pediatric patients for its high VWF:RCo:FVIII:C ratio. $^{7}$ A high ratio of VWF:RCo:FVIII:C, as is the case for both Formulation HP and Formulation V, may help patients obtain therapeutic VWF plasma levels without leading to long-term accumulation of FVIII, which is associated with an increased risk of thromboembolic events. ${ }^{19-23}$ In addition, administration of Formulation V normalized FVIII:C levels immediately following the first infusion, thus ensuring that FVIII levels were hemostatically sufficient.

The specific VWF:RCo activity varies between VWF/ FVIII concentrates, affecting the volume administered to the patient. Formulation V has the highest VWF:RCo activity/mL infused concentrate $(240 \mathrm{IU} / \mathrm{mL})$; with the lowest values for WILFACTIN $^{\circledR} /$ WILLFACT (LFB S.A., Les Ulis, France) and WILATE $^{\circledR}$ (Octapharma AG, Lachen, Switzerland; both 100 $\mathrm{IU} / \mathrm{mL}) .{ }^{10,24-26}$ Higher concentrations allow for reduction of the administered volume to the ultimate benefit of patients, especially pediatric ones.

This study included 16 evaluable patients in the efficacy population who received on-demand or prophylaxis treatment with Formulation $\mathrm{V}$ for 12 months. The overall prophylactic hemostatic efficacy and hemostatic efficacy per NSB event were assessed by the investigator as either "excellent" or "good" for all patients. Additionally, hemostatic efficacy assessed by patients/legal guardians was rated as excellent or good for $92.4 \%$ of bleeding days of all evaluated NSB events.

Prophylaxis of patients with severe VWD is challenging. Although a higher incidence of minor events occurred compared with that observed during the year before study entry, this was not unexpected in young children (the four subjects in the prophylaxis arm were 3 , 5, 5, and 8 years old). Furthermore, any comparison between NSB events before and during the study may be hampered by underreporting in the non-study period compared with the stringent reporting during the study. Importantly, a decrease in major NSB events was reported, as well as a reduction in the incidence of joint bleeds compared with on-demand treatment. This is despite the fact that three out of the four patients on prophylaxis had type 3 VWD, which is characterized by severe quantitative defects in VWF. The reduction in joint bleeds is particularly noteworthy, as it may potentially result in a lower rate of late joint complications. It is recommended that prophylaxis for joint bleeding should be started as soon as possible in patients with VWD to decrease the risk of this complication. $^{7}$ In addition, a considerable number of the minor NSB events reported during the study did not require additional Formulation $\mathrm{V}$ administration (ranging from $9.1 \%$ to $34.3 \%$ ), and fewer NSB events in the 
prophylaxis arm required treatment with more than one Formulation $\mathrm{V}$ infusion compared with the on-demand arm ( $2.2 \%$ vs $26.0 \%$, respectively). Given the positive benefits of prophylactic Formulation V, these patients continued their prophylaxis therapy in an extension study for up to an additional 28 months (NCT01224808).

Formulation $\mathrm{V}$ was well tolerated and the safety profile was consistent with that observed in previous studies involving adults and children, ${ }^{27-30}$ and of other FVIII/ VWF concentrates. ${ }^{17,31-33}$ Overall, the benefit-risk profile for Formulation $\mathrm{V}$ was favorable.

Limitations of this study include the low number of patients, the fact that none of the patients were treatmentnaïve, and the lack of patients with type 1 VWD that may limit the general applicability of the results. Genetic testing to determine the underlying VWD mutations was not carried out in this study, but the potential impact of different mutations on PK would be a worthwhile addition to future studies.

\section{Conclusion}

In summary, although the PK of Formulation V in the pediatric population was generally similar to that of the adult population, differences observed necessitate use of a higher prophylactic dose in children with severe VWD. In all patients, investigator-assessed hemostatic efficacy was excellent or good to stop an NSB event, as well as for prophylaxis, with the latter approach resulting in a reduction in the incidence of major and joint bleeds. No clinically relevant differences in PK, hemostatic efficacy, and safety were observed between patients $<6$ years and 6 to $<12$ years of age. These results provide evidence for use of Formulation $\mathrm{V}$ to treat and prevent bleeding events in pediatric patients with severe VWD, supporting the favorable benefit-risk profile of this concentrate.

\section{Abbreviations}

$\mathrm{ABR}$, annualized bleeding rate; AUC, area under the curve; bw, body weight; $C L$, total clearance; $\mathrm{C}_{\max }$, maximum plasma concentration; DDAVP, desmopressin acetate (1-deamino8-D-arginine vasopressin); FVIII, coagulation factor VIII; FVIII:C, coagulation factor VIII: coagulant activity; IR, incremental recovery; IU, international units; MRT, mean residence time; NSB, nonsurgical bleed; PK, pharmacokinetic; $t_{1 / 2}$, halflife; TEAE, treatment-emergent adverse event; $\mathrm{V}_{\mathrm{ss}}$, volume of distribution at steady state; VWD, von Willebrand disease; VWF, von Willebrand factor; VWF:Ag, von Willebrand factor: antigen; VWF:CB, von Willebrand factor: collagen binding; VWF:RCo, von Willebrand factor: ristocetin cofactor.

\section{Data Sharing Statement}

CSL will only consider requests to share Individual Patient Data (IPD) that are received from systematic review groups or bona-fide researchers. CSL will not process or act on IPD requests until 12 months after article publication on a public website. An IPD request will not be considered by CSL unless the proposed research question seeks to answer a significant and unknown medical science or patient care question. Applicable country-specific privacy and other laws and regulations will be considered and may prevent sharing of IPD.

Requests for use of the IPD will be reviewed by an internal CSL review committee. If the request is approved, and the researcher agrees to the applicable terms and conditions in a data sharing agreement, IPD that has been appropriately anonymized will be made available. Supporting documents including study protocol and Statistical Analysis Plan will also be provided.

For information on the process and requirements for submitting a voluntary data sharing request for IPD, please contact CSL at clinicaltrials@cslbehring.com.

\section{Acknowledgments}

Editorial support (medical writing and editing assistance, collation of author comments and manuscript formatting) was provided by Susan Brackenridge $\mathrm{PhD}$, Fishawack Communications Ltd, and was funded by CSL Behring, Germany.

\section{Author Contributions}

All authors made substantial contributions to study design or data acquisition, were involved in drafting or revising the manuscript, provided approval of the final version, and agree to be accountable for all aspects of the work.

\section{Funding}

This study was supported by a grant from CSL Behring, which was responsible for operation of the trial, including study design, data collection and data analysis.

\section{Disclosure}

GA has received reimbursements for attending symposia/ congresses and/or honoraria for speaking and/or consulting and/or funds for research support from CSL Behring.

OS has received honoraria from Novo Nordisk, Takeda, and CSL Behring, and declares membership of speaker bureaus for Novo Nordisk, Takeda and Pfizer. 
WS and TR are employees of CSL Behring. The authors report no other conflicts of interest in this work.

\section{References}

1. Barr RD, Sek J, Horsman J, et al. Health status and health-related quality of life associated with von Willebrand disease. Am J Hematol. 2003;73(2):108-114. doi:10.1002/ajh.10327

2. Nichols WL, Hultin MB, James AH, et al. von Willebrand disease (VWD): evidence-based diagnosis and management guidelines, the National Heart, Lung, and Blood Institute (NHLBI) Expert Panel report (USA). Haemophilia. 2008;14(2):171-232. doi:10.1111/ j.1365-2516.2007.01643.x

3. Flood VH, Montgomery RR. von Willebrand disease: biologic diagnosis. In: Lee CA, Berntorp EE, Hoots WK, editors. Textbook of Hemophilia. Oxford, UK: Wiley-Blackwell; 2010:294-301.

4. Heijdra JM, Cnossen MH, Leebeek FWG. Current and emerging options for the management of inherited von Willebrand disease. Drugs. 2017;77(14):1531-1547. doi:10.1007/s40265-017-0793-2

5. Sadler JE, Budde U, Eikenboom JC, et al. Update on the pathophysiology and classification of von Willebrand disease: a report of the subcommittee on von Willebrand Factor. J Thromb Haemost. 2006;4 (10):2103-2114. doi:10.1111/j.1538-7836.2006.02146.x

6. Mannucci PM. How I treat patients with von Willebrand disease. Blood. 2001;97(7):1915-1919. doi:10.1182/blood.V97.7.1915

7. Castaman G, Linari S. Human von Willebrand factor/factor VIII concentrates in the management of pediatric patients with von Willebrand disease/hemophilia A. Ther Clin Risk Manag. 2016;12:1029-1037. doi:10.2147/TCRM.S87543

8. Federici AB, Mannucci PM. Diagnosis and management of von Willebrand disease. Haemophilia. 1999;5(Suppl 2):28-37. doi:10.10 46/j.1365-2516.1999.0050s2028.x

9. Laffan MA, Lester W, O'Donnell JS, et al. The diagnosis and management of von Willebrand disease: a United Kingdom Haemophilia centre doctors organization guideline approved by the British committee for standards in haematology. Br J Haematol. 2014;167 (4):453-465. doi:10.1111/bjh.13064

10. CSL Behring. VONCENTO ${ }^{\circledR}$ Summary of Product Characteristics. 2018. Available from: https://www.medicines.org.uk/emc/product/ 5341/. Accessed June 22, 2018.

11. Lissitchkov TJ, Buevich E, Kuliczkowski K, et al. Pharmacokinetics, efficacy, and safety of a plasma-derived VWF/FVIII concentrate (VONCENTO) for on-demand and prophylactic treatment in patients with von Willebrand disease (SWIFT-VWD study). Blood Coagul Fibrinolysis. 2017;28(2):152-162. doi:10.1097/MBC.000000000000 0568

12. Harper P, Favaloro EJ, Curtin J, Barnes C, Dunkley S. Human plasma-derived FVIII/VWD concentrate (Biostate): a review of experimental and clinical pharmacokinetic, efficacy and safety data. Drugs Context. 2016;5:212292. doi:10.7573/dic.212292

13. Skotnicki A, Lissitchkov TJ, Mamonov V, et al. Efficacy, safety and pharmacokinetic profiles of a plasma-derived VWF/FVIII concentrate (VONCENTO(R)) in subjects with haemophilia A (SWIFT-HA study). Thromb Res. 2016;137:119-125. doi:10.1016/j.thromres.20 15.10 .014

14. EMA guideline on the clinical investigation of human plasma derived von Willebrand factor products (CPMP/BPWG/220/02). Available from: http://www.ema.europa.eu/docs/en_GB/document_library/ Scientific_guideline/2010/01/WC500067126.pdf. Accessed April 17, 2020.

15. Federici AB, Castaman G, Franchini M, et al. Clinical use of haemate $P$ in inherited von Willebrand's disease: a cohort study on 100 Italian patients. Haematologica. 2007;92(7):944-951. doi:10.3324/haematol. 11124
16. Windyga J, von Depka-prondzinski M, European Wilate Study G. Efficacy and safety of a new generation von Willebrand factor/factor VIII concentrate (Wilate(R)) in the management of perioperative haemostasis in von Willebrand disease patients undergoing surgery. Thromb Haemost. 2011;105(6):1072-1079. doi:10.1160/TH10-100631

17. Borel-Derlon A, Federici AB, Roussel-Robert V, et al. Treatment of severe von Willebrand disease with a high-purity von Willebrand factor concentrate (Wilfactin): a prospective study of 50 patients. J Thromb Haemost. 2007;5(6):1115-1124. doi:10.1111/j.1538-7836. 2007.02562.x

18. Shire. VONVENDI ${ }^{\circledR}$ Prescribing Information. 2018. Available from: https://www.shirecontent.com/PI/PDFs/VONVENDI_USA_ENG. pdf. Accessed June 22, 2018.

19. Coppola A, Franchini M, Makris M, Santagostino E, Di Minno G, Mannucci PM. Thrombotic adverse events to coagulation factor concentrates for treatment of patients with haemophilia and von Willebrand disease: a systematic review of prospective studies. Haemophilia. 2012;18(3):e173-e187. doi:10.1111/j.1365-2516.2012. 02758.x

20. Koster T, Blann AD, Briet E, Vandenbroucke JP, Rosendaal FR. Role of clotting factor VIII in effect of von Willebrand factor on occurrence of deep-vein thrombosis. Lancet. 1995;345(8943):152-155. doi:10.1016/S0140-6736(95)90166-3

21. Makris M, Colvin B, Gupta V, Shields ML, Smith MP. Venous thrombosis following the use of intermediate purity FVIII concentrate to treat patients with von Willebrand's disease. Thromb Haemost. 2002;88(3):387-388. doi:10.1055/s-0037-1613227

22. Mannucci PM. Venous thromboembolism in von Willebrand disease. Thromb Haemost. 2002;88(3):378-379. doi:10.1055/s-0037-1613225

23. Mannucci PM, Chediak J, Hanna W, et al. Treatment of von Willebrand disease with a high-purity factor VIII/von Willebrand factor concentrate: a prospective, multicenter study. Blood. 2002;99 (2):450-456. doi:10.1182/blood.V99.2.450

24. CSL Behring. HAEMATE $\mathrm{P}^{\circledR}$ summary of product characteristics. 2018. Available from: https://www.medicines.org.uk/emc/medicine/ 19412. Accessed June 22, 2018.

25. LFB Biopharmaceuticals. WILLFACT ${ }^{\circledR}$ summary of product characteristics. 2016. Available from: https://www.medicines.org.uk/emc/ medicine/29947. Accessed June 22, 2018.

26. Octapharma. WILATE ${ }^{\circledR}$ summary of product characteristics. 2014. Available from: https://www.medicines.org.uk/emc/medicine/27202. Accessed June 22, 2018.

27. Favaloro EJ, Lloyd J, Rowell J, et al. Comparison of the pharmacokinetics of two von Willebrand factor concentrates [BiostateandAHF(HighPurity)] in people with von Willebrand disorder. A randomised cross-over, multi-centre study. Thromb Haemost. 2007;97(6):922-930. doi:10.1160/TH06-09-0495

28. Dunkley S, Baker RI, Pidcock M, et al. Clinical efficacy and safety of the factor VIII/von Willebrand factor concentrate BIOSTATE in patients with von Willebrand's disease: a prospective multi-centre study. Haemophilia. 2010;16(4):615-624. doi:10.1111/j.1365-2516.2010.02206.x

29. Shortt J, Dunkley S, Rickard K, Baker R, Street A. Efficacy and safety of a high purity, double virus inactivated factor VIII/von Willebrand factor concentrate (Biostate) in patients with von Willebrand disorder requiring invasive or surgical procedures. Haemophilia. 2007;13(2):144-148. doi:10.1111/j.1365-2516.2006.01430.x

30. Howman R, Barnes C, Curtin J, et al. The clinical efficacy and safety of the FVIII/VWF concentrate, BIOSTATE(R), in children with von Willebrand disorder: a multi-centre retrospective review. Haemophilia. 2011;17(3):463-469. doi:10.1111/j.1365-2516.2010. 02445.x

31. Berntorp E, Archey W, Auerswald G, et al. A systematic overview of the first pasteurised VWF/FVIII medicinal product, Haemate $\mathrm{P} /$ Humate -P: history and clinical performance. Eur $J$ Haematol Suppl. 2008;70:3-35. doi:10.1111/j.1600-0609.2008.01049.x 
32. Berntorp E, Windyga J, European Wilate Study Group. Treatment and prevention of acute bleedings in von Willebrand disease-efficacy and safety of Wilate, a new generation von Willebrand factor/factor VIII concentrate. Haemophilia. 2009;15(1):122-130. doi:10.1111/ j.1365-2516.2008.01901.x
33. Federici AB, James P. Current management of patients with severe von Willebrand disease type 3: a 2012 update. Acta Haematol. 2012;128(2):88-99. doi:10.1159/000338208

\section{Publish your work in this journal}

The Journal of Blood Medicine is an international, peer-reviewed, open access, online journal publishing laboratory, experimental and clinical aspects of all aspect pertaining to blood based medicine including but not limited to: Transfusion Medicine; Blood collection, Donor issues, Transmittable diseases, and Blood banking logistics; Immunohematology; Artificial and alternative blood based

Submit your manuscript here: http://www.dovepress.com/journal-of-blood-medicine-journa therapeutics; Hematology; Biotechnology/nanotechnology of blood related medicine; Legal aspects of blood medicine; Historical perspectives. The manuscript management system is completely online and includes a very quick and fair peer-review system. Visit http://www.dovepress.com/testimonials.php to read real quotes from published authors. 